\title{
Erratum: Size dependence of the surface tension of a free surface of an isotropic fluid [Phys. Rev. E 95, 062801 (2017)]
}

\author{
Sergii Burian ๑, Mykola Isaiev, Konstantinos Termentzidis, Vladimir Sysoev, and Leonid Bulavin
}

(Q) (Received 12 June 2020; published 24 June 2020)

DOI: 10.1103/PhysRevE.101.069903

There was a misprint in Eq. (3) of our paper. The correct Eq. (3) should read as follows:

$$
\frac{\sigma_{s}(R)}{\sigma^{\infty}}=\left|\frac{z}{z-\delta / R}\right|^{\left(3+3 z+z^{2}\right) / 3(1+z)^{2}}\left(\frac{x^{2}+y^{2}}{(x+\delta / R)^{2}+y^{2}}\right)^{a} e^{2 b\{\arctan (x / y)-\arctan [(x+\delta / R) / y]\}},
$$

where $x=1-1 / 2 \sqrt[3]{2}, y=\sqrt{3} / 2 \sqrt[3]{2}, z=-1-1 / \sqrt[3]{2}, a=(2-\sqrt[3]{2})(1+\sqrt[3]{2}) / 6$, and $b=(1+\sqrt[3]{2}) / \sqrt[3]{4} \sqrt{3}$.

This misprint does not impact the presented results and conclusions; all data in the paper have been calculated and analyzed based on the correct equation.

We would like to thank Professor S. Sh. Rekhviashvili for pointing out this typographical error in his paper [1].

[1] S. S. Rekhviashvili, Colloid J. 82, 342 (2020). 